\title{
CONSTRUCCIÓN Y CALIBRACIÓN DE UNA CELDA DE SEDIMENTACIÓN GRAVIMÉTRICA PARA LA MEDICIÓN FOTOMÉTRICA DE LA DISTRIBUCIÓN DE TAMAÑO DE MICROPARTÍCULAS
}

\author{
Gonzalo Salinas-Salas ${ }^{1} \quad$ Michael Stintz $^{2} \quad$ Benno Wessely $^{2}$ \\ Recibido el 18 de noviembre de 2004, aceptado el 24 de mayo de 2005
}

\begin{abstract}
RESUMEN
El presente trabajo muestra la base teórica, el desarrollo, la implementación y los resultados alcanzados al emplearse una celda de sedimentación gravimétrica para la detección de la distribución de tamaño o diámetro medio de un conjunto de micropartículas, a partir de su detección a través de un sistema de tipo óptico, basado en la medición de la extinción o decaimiento de la intensidad de flujo de fotones de un haz de luz que atraviesa una suspensión compuesta por un material particulado y agua. La celda fue construida de manera modular, mientras que su calibración y los ensayos se realizaron utilizando suspensiones compuestas por partículas de tamaño estandarizado de dióxido de silicio amorfo (Geltech $\mathrm{S}$ 1500) de un diámetro medio nominal de $1,5 \mu \mathrm{m}$ en agua. Los resultados alcanzados mostraron que el uso de esta celda de sedimentación es en extremo conveniente, ya que fue posible detectar la distribución de tamaño de micropartículas con precisión y a un relativo bajo costo, lo que permitiría aplicarlo ventajosamente en la industria de procesos en general.
\end{abstract}

Palabras clave: Sedimentación, medición, fotométrica, tamaño, micropartículas.

\section{ABSTRACT}

This paper present the theoretical basis, development, implementation and results of from a gravimetric sedimentation cell for determination of size distribution and median diameter of a sample of micro particles. The measurement is based on the transmittance of light through a suspension particles in water. The cell was built in modular form and was calibrated with suspensions of amorphous silicion dioxid with a median diameter of $1.5 \mu$ m. The results indicate that the sedimentation cell is very simple to use and that the size distribution of micro particles can be determined at low cost. This procedure may be conveniently applied in the many industrials processes.

Keywords: Sedimentation, measurement, photometric, size, micro particles.

\section{INTRODUCCIÓN}

La determinación de la distribución de tamaño de micropartículas es un problema que está adquiriendo cada vez una importancia mayor para la industria en general y la de procesos en particular, ya que impacta directamente en las tecnologías de separación de fase y en especial en las tecnologías medioambientales, ya que en la práctica no existe un tamaño o diámetro único de partícula, sino que un tamaño o diámetro que pretende representar un conjunto de partículas de dimensiones similares, lo que implica que para la misma medida de tamaño nominal existe un porcentaje con un tamaño mayor y otro con un tamaño menor al valor nominal.
Actualmente existen diversas técnicas para la determinación de la distribución de tamaño de micropartículas, las que tienen como base las técnicas denominadas como:

- Incremental

- Acumulativa

La técnica incremental se caracteriza por medir la concentración del material particulado que existe $y / 0$ atraviesa por una determinada posición espacial de la celda de medida. La técnica acumulativa, en cambio, mide la masa o el volumen total de material particulado contenido dentro de la celda de medida. Cabe considerar

\footnotetext{
1 Universidad de Talca, Departamento de Tecnologías Industriales, gsalinas@utalca.cl

2 Technische Universität Dresden, Institut für Verfahrenstechnik und Umwelttechnik, mechanvt@rcs.urz.tu-dresden.de
} 
que estas técnicas se pueden aplicar tanto cuando la celda de medida y por ende, las micropartículas que contiene, se encuentran bajo la acción del campo de fuerza gravitatorio o uno de tipo centrífugo [3] y [5].

Los principios físicos de las tecnologías para la medición de la densidad de distribución de tamaño de micropartículas permiten agrupar a estas tecnologías de la manera siguiente:

a) Medición a partir de fuerzas gravimétricas que actúan sobre el conjunto de partículas.

- Método de la pipeta

- Método por decantación

- Método por balanza de sedimentación

b) Medición a partir de la absorción de radiaciones electromagnéticas por el conjunto de partículas.

- Método de extinción fotométrica

- Método de difracción fotométrica

- Método de absorción de rayos X

c) Medición por cambios de densidad de la suspensión formada por fluido y el conjunto de partículas.

- Método manométrico

- Método por intercambio de cuerpos

d) Medición a través del debilitamiento por absorción de radiación $\beta$ por el conjunto de partículas.

Cada una de las tecnologías ya mencionadas ofrece ventajas y desventajas para una situación específica, de modo que su aplicación se realiza de acuerdo a las características del material que se desea medir, el grado de precisión de la medida que se desea alcanzar y la norma de ingeniería bajo la cual se realiza el ensayo [2].

Dado que esta tecnología es compleja de desarrollarse por la elevada precisión que demanda la medición de micropartículas, esto se traduce en los elevados costos que tienen los equipos de medida. Por otra parte, esta tecnología se desarrolla fuera del país, lo que encarece aún más los equipos, impidiendo con ello el acceso a las pequeñas y medianas empresas a estos aparatos que mejorarían la calidad de sus productos. De ahí que el presente trabajo se centra en el desarrollo de una celda de sedimentación gravimétrica cuyas principales características son su bajo costo, su precisión, el hecho que pueda ser construida en el país y que utiliza como sistema de detección el método de extinción fotométrica de un haz de luz visible, para determinar la distribución de tamaño de partículas, en el límite de la aplicabilidad que tiene la metodología de medición a través de la sedimentación gravimétrica, ya que la literatura indica que esta metodología es aplicable para la determinación de la distribución de tamaño de partículas cuyo diámetro medio sea mayor a $1 \mu \mathrm{m}$. La razón de este límite está en el hecho de que las partículas de menor tamaño al ya indicado tienen una masa muy pequeña, de modo que la acción del campo gravitatorio sobre ellas es tan débil que impide su sedimentación, ya que ellas permanecen en suspensión por efecto de los flujos convectivos del fluido que tienen lugar en el interior de la cubeta y por la acción de fuerzas electroquímicas que se presentan en la superficie de las micropartículas haciendo que éstas interactúen entre sí [2], [5] y [8].

Por lo anterior y a fin de que la fuerza dominante en el proceso de sedimentación fuera la gravimétrica, es que se utilizó como material de prueba a partículas calibradas de silicio amorfo $\left(\mathrm{SiO}_{2}\right)$ rígidas de forma esférica y con diámetro medio nominal de $1,5 \mu \mathrm{m}$, las que fueron suspendidas en agua con un índice de acidez pH 9,2.

\section{FUNDAMENTACIÓN TEÓRICA}

\section{Aspectos hidrodinámicos}

La sedimentación de partículas en un fluido no es un fenómeno nuevo, ya que ha sido utilizada con fines prácticos desde épocas muy remotas por la humanidad y actualmente juega un rol de especial relevancia para las tecnologías de descontaminación, en la industria farmacéutica, la de procesos, en la elaboración de revestimientos de todo tipo, de materiales cerámicos de alta resistencia y otras aplicaciones.

Como fue indicado, en términos fisicoquímicos en un proceso de sedimentación gravimétrica existen dos tipos de fuerzas, las que corresponden a las fuerzas mecánicas originadas por la acción de un campo de fuerzas gravimétrico o centrífugo y las fuerzas de tipo electroquímico que se dan por la acción de las diferencias de potencial electrostático entre las superficies de las partículas, las fuerzas de Van der Waals y otras; a todas estas fuerzas de origen electroquímico se les denominan como fuerzas débiles y son modeladas a través de la Teoría DLVO, desarrollada por Derjarin, Landau, Verwey y Overbeck [2].

Considerando una sedimentación de micropartículas en que la fuerza dominante es generada por el campo gravitatorio, es posible modelar el comportamiento de una partícula rígida, de forma esférica, la que se sumerge en un fluido viscoso desde una posición dada con una velocidad inicial nula, al aplicarse a ésta la ecuación de 
D'Alambert, considerando sólo los términos de cambio de cantidad de movimiento, el peso de la partícula, la fuerza de flotación y la de arrastre viscoso que se ejercen sobre la partícula, lo que da lugar a la ecuación que sigue, donde $\mathrm{z}$ representa a la posición de la micropartícula en el sentido de la aceleración de gravedad [9]:

$$
m_{p} \cdot \frac{d^{2} \vec{z}(t)}{d t}=\vec{W}_{\text {particula }}-\vec{F}_{\text {flotación }}-\vec{F}_{\text {arrastre }}
$$

Como todo movimiento de un sólido, en este caso una partícula esférica en un medio viscoso, presenta una primera fase de movimiento, en que existe una desaceleración y por lo tanto la velocidad de la partícula es variable con respecto del tiempo y la posición. A medida que la partícula se sumerge en el fluido, ésta tiende a un estado de equilibrio estático, donde el peso de la partícula es compensado por la acción de las fuerzas de flotación y arrastre, de modo que la partícula alcanza una velocidad de sumergimiento constante a la que se le denomina velocidad terminal.

El problema de evaluar matemáticamente la solución de la ecuación (1) fue resuelto analíticamente por Stokes. Sin embargo, esta solución tiene una muy importante restricción, la que se refiere al régimen de escurrimiento del fluido por sobre la esfera debe no sólo ser laminar, sino que además su Número de Reynolds debe ser menor a 0,25 , a fin de asegurar la exactitud en el valor del coeficiente de arrastre para una esfera rígida [9], [10].

Considerando que el Número de Reynolds que se presenta sobre la partícula esférica no es mayor a 0,25 , se puede obtener la siguiente solución genérica para la ecuación diferencial (1), entendiendo que la dirección de movimiento es según la dirección del campo gravitatorio.

$$
\frac{d^{2} \vec{z}(t)}{d t^{2}}=\frac{\left(\rho_{p}-\rho_{f}\right) \cdot \vec{g}}{\rho_{p}} \cdot e^{-\frac{3 \cdot \pi \cdot x \cdot \mu}{m_{p}} \cdot t}
$$

Donde:

$$
\begin{array}{ll}
\frac{d^{2} \vec{z}(t)}{d t^{2}} & : \text { Aceleración de la partícula } \\
\rho_{p} & \text { : Densidad media de la partícula } \\
\rho_{f} & \text { : Densidad media del fluido } \\
m_{p} & \text { : Masa de la partícula } \\
g & \text { : Aceleración de gravedad } \\
\mu & \text { : Viscosidad dinámica del fluido } \\
t & \text { : Tiempo }
\end{array}
$$

Integrando la función aceleración de la micropartícula es posible obtener la expresión de la velocidad de ésta en función del tiempo.

$$
\frac{d \vec{z}(t)}{d t}=\frac{\left(\rho_{p}-\rho_{f}\right) \cdot m_{p} \cdot \vec{g}}{3 \cdot \pi \cdot x \cdot \mu \cdot \rho_{p}} \cdot\left(1-e^{-\frac{3 \cdot \pi \cdot x \cdot \mu}{m_{p}} \cdot t}\right)
$$

Donde:

$$
\frac{d \vec{z}(t)}{d t} \text { : Velocidad de sedimentación de la partícula }
$$

Siendo la posición de la partícula evaluable a partir de la siguiente ecuación:

$$
\vec{z}(t)=\frac{\left(\rho_{p}-\rho_{f}\right) \cdot m_{p} \cdot \vec{g}}{3 \cdot \pi \cdot x \cdot \mu \cdot \rho_{p}}\left(t+\frac{m_{p}}{3 \cdot \pi \cdot x \cdot \mu} \cdot e^{-\frac{3 \cdot \pi \cdot x \cdot \mu}{m_{p}} \cdot t}\right)
$$

Donde:

$z(t) \quad$ : Posición de la partícula medida desde su posición inicial de sedimentación en el sentido del campo gravitatorio

Considerando un tiempo de sumergimiento que tienda al infinito, la expresión anterior permite determinar la velocidad de sumergimiento terminal de la partícula, a la que se le denomina velocidad de Stokes, la que adopta la forma siguiente y tiene como dirección la del campo gravitatorio:

$$
v_{S t}=\frac{\left(\rho_{p}-\rho_{f}\right) \cdot x^{2} \cdot g}{18 \cdot \mu}
$$

Donde:

$v_{S t}:$ Velocidad de Stokes

Esta velocidad en el caso de conjuntos o colectivos de partículas se ve influenciada por la interacción que se genera entre las partículas, las que pueden manifestar a través de choques entre partículas, las interacciones hidrodinámicas entre éstas, el arrastre engendrado por la turbulencia provocada por la sedimentación dentro del recipiente donde ésta ocurre, a las que se suman las fuerzas electroquímicas superficiales.

Estos efectos, dependiendo de la concentración de material particulado tienden a disminuir la velocidad de sedimentación, de ahí la importancia de su evaluación. Estos efectos se han analizado desde una perspectiva de carácter netamente empírico por lo complejo de los 
fenómenos involucrados, lo que ha dado lugar a la aparición de diversas funciones de corrección a la velocidad de Stokes, a fin de obtener una velocidad más representativa del fenómeno al asociarlo con la concentración volumétrica de partículas presentes en la suspensión. La manera usual de utilizar estas funciones de corrección es la siguiente [1-4].

$$
v=f\left(C_{v}\right) \cdot v_{S t}
$$

Donde:

$v \quad:$ Velocidad de sedimentación terminal de un colectivo de partículas

$f\left(C_{v}\right)$ : Función corrección de velocidad de sedimentación terminal o de Stokes

Por lo general, estas funciones de corrección están asociadas no sólo a la concentración volumétrica de las partículas presentes en la suspensión, sino que además consideran la viscosidad del fluido en el que las partículas están dispersas. De modo que la forma final que toma la función corrección es la siguiente:

$$
f\left(C_{v}\right)=\frac{\left(1-C_{v}\right)^{2}}{\mu\left(C_{v}\right)}
$$

Donde:

$\mu\left(C_{v}\right)$ : Función corrección de viscosidad de la suspensión

Las funciones de corrección de viscosidad que más comúnmente son empleadas para suspensiones con concentraciones pequeñas de material particulado, son las propuestas por Einstein y Batchelor [4] y [1]. Estas formas se presentan en el mismo orden a continuación:

$$
\begin{aligned}
& f\left(C_{v}\right)=\left(1+2,5 \cdot C_{v}\right) \\
& f\left(C_{v}\right)=\left(1-C_{v}\right)^{-4,55}
\end{aligned}
$$

De lo anteriormente expuesto se permite concluir que a partir de la aplicación de la siguiente ecuación se puede determinar el diámetro medio de un colectivo de partículas esféricas rígidas que se sumergen en un fluido viscoso, con velocidad constante que da lugar a un Número de Reynolds menor a 0,25.

Si se considera el caso de partículas que sean de forma no esférica, independientemente que su geometría sea simétrica o asimétrica, se utiliza el concepto de factor de esfericidad, el que se define como la división entre el área de una esfera cuyo volumen sea idéntico al de la partícula a medir y el área superficial de ésta. Por extensión del concepto anterior, el factor de esfericidad puede también definirse como el cuadrado de la división entre el diámetro de una esfera de volumen idéntico y el diámetro de una esfera de área idéntica al de la partícula [2], [3], [7] y [8].

$$
k_{\psi}=\frac{A_{0 K}}{A_{0 P}}=\left(\frac{x_{V}}{x_{A 0}}\right)^{2}
$$

Donde:

$k \psi \quad: \quad$ Factor de esfericidad

$A_{0 K} \quad$ : Área de una esfera de volumen idéntico al de la partícula

$A_{O P} \quad$ : Área efectiva de la partícula

$x_{V} \quad$ : Diámetro de una esfera de volumen idéntico

$x_{A 0}$ : Diámetro de una esfera de área idéntica

A partir de esta relación se plantea el denominado factor dinámico de forma, que se define como la división entre la velocidad estacionaria real de sedimentación de la partícula y la velocidad de sedimentación estacionaria de una partícula esférica cuyo volumen sea idéntico al de la partícula asimétrica. En el caso de que durante la fase de velocidad de sedimentación estacionaria se presente un Número de Reynolds menor a 0,5, el factor de corrección de la velocidad de sedimentación por efecto de la geometría de la partícula pasa a ser la raíz cuadrada del factor de esfericidad y la división entre la velocidad de Stokes de la partícula y la velocidad de Stokes de una partícula esférica de volumen idéntico [2], [3].

$$
K_{\varphi}=\sqrt{k_{\psi}}=\frac{v_{S t, p}}{v_{S t, e}}
$$

Donde:

$$
\begin{array}{ll}
K \varphi & : \text { Factor de corrección } \\
v_{S t, p}: \text { Velocidad de Stokes de la partícula } \\
v_{S t, e}: \text { Velocidad de Stokes de una partícula esférica } \\
& \text { de volumen idéntico }
\end{array}
$$

Luego, para la representación de la distribución de tamaño de partícula, se utiliza el diámetro de la partícula esférica de volumen idéntico, el que se evalúa a partir de la siguiente ecuación:

$$
x=\sqrt{\frac{1}{K_{\varphi}} \cdot \frac{18 \cdot \mu \cdot v_{S t}}{f\left(C_{v}\right) \cdot\left(\rho_{p}-\rho_{f}\right) \cdot g}}
$$




\section{Aspectos ópticos}

El principio físico en que se basa la determinación del tamaño de partículas tiene como fundamento la reducción que sufre la intensidad luminosa de un haz de luz que atraviesa por un espesor determinado de una suspensión que se encuentra contenida en una cubeta. De manera que es posible relacionar este decaimiento de la intensidad luminosa con el tamaño de las partículas presentes en la suspensión, lo que es representado por el denominado volumen específico de área de extinción, su concentración volumétrica y el espesor que debe atravesar el haz de luz.

Si se considera un espesor de suspensión constante y un tamaño dado de partícula, se puede plantear el comportamiento del logaritmo natural de la división entre la intensidad luminosa que logra atravesar la suspensión respecto de la intensidad luminosa que atraviesa al fluido cuando en éste no se encuentran partículas suspendidas, respecto de la concentración volumétrica de la suspensión. A la división entre las intensidades lumínicas ya indicadas se denomina transmisión $(\mathrm{T})$.

Este comportamiento para suspensiones diluidas es lineal, para luego a medida que la suspensión se concentra comienza a comportarse asintóticamente. Este comportamiento se debe al hecho de que a medida que la suspensión es más concentrada, comienza a generarse interferencia óptica entre las sombras que proyectan las partículas.

Por lo anterior, se desprende que este procedimiento sólo puede aplicarse a suspensiones diluidas, donde el comportamiento del logaritmo natural de la transmisión sea lineal respecto de la concentración volumétrica.

Este comportamiento es modelado a través de la Ley de Lambert-Beer [2], la que indica.

$$
\ln T=\ln \frac{I}{I_{0}}=-A_{p} \cdot C_{v} \cdot L
$$

Donde:

$T \quad:$ Transmisión

I : Intensidad lumínica captada por el sensor en presencia de material particulado en la suspensión

$I_{0} \quad$ : Intensidad lumínica captada por el sensor sin material particulado en la suspensión

$A_{p} \quad:$ Área específica de extinción

$L^{p} \quad$ : Ancho de la cubeta que contiene a la suspensión
Por su parte, en el caso de un colectivo de partículas el área específica de extinción queda definido como sigue:

$$
A_{p}=\frac{3}{2} \cdot \frac{K(x)}{x}=\frac{E / L}{C_{v}}
$$

Donde:

$K(x) \quad$ : Función coeficiente de extinción E : Extinción

La función coeficiente de extinción es característica no sólo para un determinado tipo de partícula y de fluido, las que dan lugar a ciertas constantes ópticas, sino que también depende de la longitud de la onda incidente sobre la partícula y del ángulo de abertura o diafragma del aparato óptico que se utilice para sensar la intensidad lumínica que logra atravesar la suspensión. En todo caso, siempre se utiliza una longitud de onda incidente $(\lambda)$ menor al diámetro medio de la partícula (x).

La estimación del coeficiente de extinción $\mathrm{K}(\mathrm{x})$, para un determinado diámetro de partícula, se puede realizar tanto a partir de cálculos utilizando la teoría Mie, [2] o experimentalmente [6], siendo este último caso el más utilizado en términos prácticos.

En el caso de un colectivo de partículas de diferente diámetro, la Ley de Lambert-Beer adopta la forma siguiente:

$$
\ln \frac{I}{I_{0}}=-\frac{3}{2} \cdot C_{v 0} \cdot L \cdot \int_{0}^{x_{t}} \frac{K(x)}{x} \cdot q_{3}(x) \cdot d x
$$

Donde:

$C_{v 0} \quad: \quad$ Concentración volumétrica inicial de material particulado presente en la suspensión

$q_{3}(x)$ : Concentración incremental del tamaño de las partículas presentes en la suspensión

Siendo la concentración incremental del tamaño de las partículas presentes en la suspensión definida como el conjunto de las fracciones de masa respecto de la masa total de partículas, de aquel grupo o familia que poseen un diámetro que se encuentra dentro de dos límites definidos. Lo anterior permite la construcción de un histograma donde se representa la importancia relativa de las partículas dentro de un intervalo de diámetros dados respecto del total. 
Este diagrama permite caracterizar a un colectivo de partículas y obtener la distribución acumulativa de éstas a partir de la siguiente ecuación:

$$
Q_{3}(x)=\int_{x_{0}}^{x_{n}} q_{3}(x) d x
$$

El diagrama acumulativo presenta la ventaja que permite visualizar la fracción del total de partículas que se encuentra por sobre o bajo de un determinado tamaño o diámetro.

En el caso que no fuese posible medir inicialmente la fracción del total del número de partículas presentes en una determinada suspensión, se debe considerar que este método es de carácter óptico, por lo tanto, siempre es posible realizar diluciones de la suspensión, las que permiten al dispositivo sensar el paso de las partículas sin interferencias y por lo tanto realizar su conteo.

\section{IMPLEMENTACIÓN Y ENSAYOS}

La celda de sedimentación fue construida mediante módulos de acero, los cuales permiten montar una fuente emisora de radiación electromagnética o luz, en este caso un emisor láser, en una torre fijándolo a una altura determinada, la que se alínea mediante barras a la posición de la cubeta que contiene la suspensión y al sensor óptico que medirá el paso de luz a través de las micropartículas, evaluándose así el cambio de la extinción. Este cambio es registrado en forma de una tensión eléctrica continua, la que es medida en forma discreta cada $100 \mathrm{~s}$, siendo transmitida a un PC, que registra los datos obtenidos y puede utilizarse para la evaluación de las distribuciones de tamaño de micropartícula. La cubeta de medida fue montada en una torre central y se fijó a ésta mediante dos prensas, las que a su vez se deslizaban por las barras de alineamiento, éstas soportaban también al filtro de luz. Por su lado, la suspensión fue ingresada a la cubeta mediante bombeo.

Dado que la celda de sedimentación se basa en un proceso de sedimentación de tipo estacionario, en el cual las micropartículas se sumergirán en un fluido newtoniano, con un campo de velocidades consistentes con la solución de Stokes para el valor de la fuerza de arrastre, hace que sea conveniente determinar el tiempo que requieren las micropartículas en alcanzar la velocidad de sumergimiento terminal o velocidad de Stokes y su posición respecto de la superficie libre de la suspensión.
Considerando las características de las micropartículas y del agua utilizada, las que se indican en la sección de caracterización de materiales, y aplicando estos valores a la ecuación $\mathrm{N}^{\circ} 3$, se puede construir el gráfico siguiente, donde se muestra la función velocidad de sumergimiento de la micropartícula respecto del tiempo, el que muestra un proceso de aceleración continua que tiende a alcanzar un valor estacionario, el que corresponde a la velocidad terminal o velocidad de Stokes, la que se alcanza en un tiempo de $216,85 \mu \mathrm{s}$.

Para fines de presentar en forma más clara el fenómeno de aceleración, el gráfico $\mathrm{N}^{\circ} 1$ muestra sólo los primeros $6 \mu$ s del proceso de sedimentación.

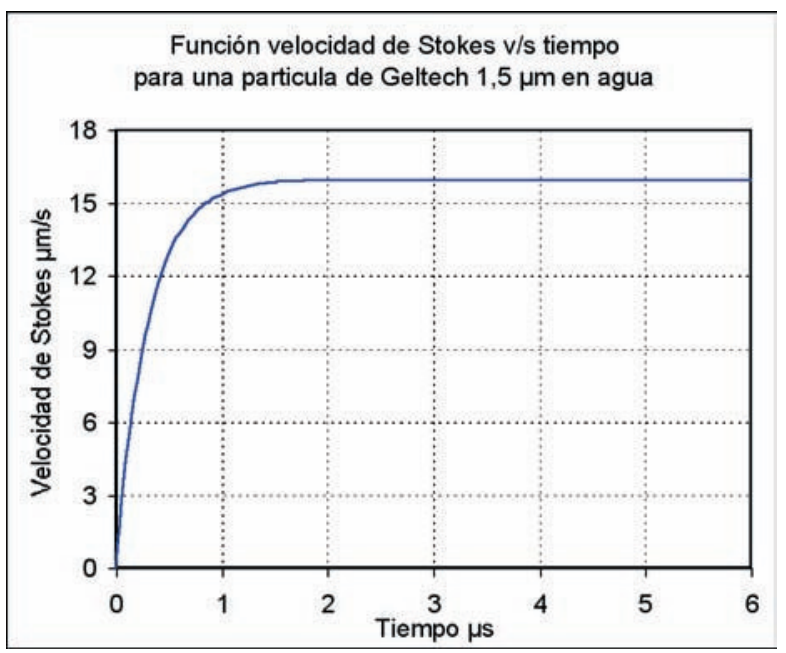

Gráfico 1 Velocidad de Stokes v/s tiempo.

Por su lado, la distancia vertical donde se puede asumir que el proceso de movimiento acelerado se transforma en movimiento constante, es posible determinarla a partir de la ecuación $\mathrm{N}^{\circ} 3$ y en este caso en particular alcanzó a $346,38 \mathrm{pm}$.

De lo anterior se desprende que para esta partícula se puede asumir que su velocidad de sedimentación será constante e igual a la velocidad de Stokes, durante la sedimentación, lo que permite ubicar la posición respecto de la superficie libre de la suspensión contenida en la cubeta que tendría tanto el láser como el lector, la que obviamente debe ser mucho mayor a 346,38 pm. En el caso particular de esta celda de sedimentación se fijó la unidad de emisor-sensor en una posición de $15 \mathrm{~mm}$ bajo la superficie libre.

Esquemáticamente es posible representar la celda de sedimentación en condiciones de operación a través de los croquis que presentan la elevación y la planta del equipo y que aparecen en las figuras $\mathrm{N}^{\circ} 1$ y $\mathrm{N}^{\circ} 2$ : 


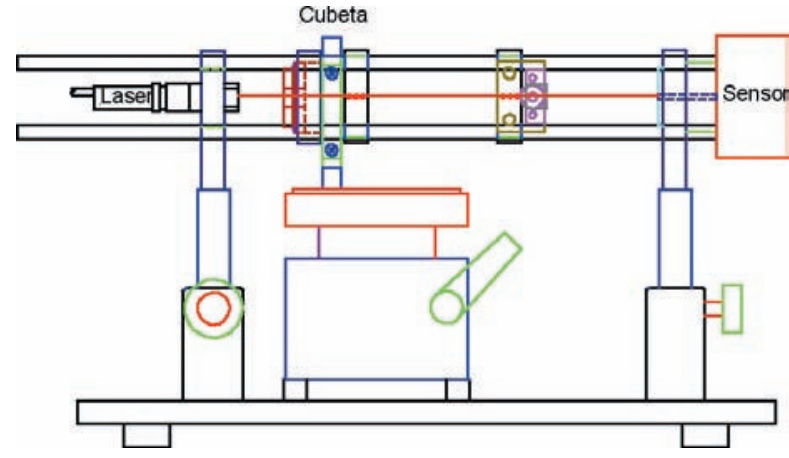

Fig. 1 Elevación de la celda de sedimentación.

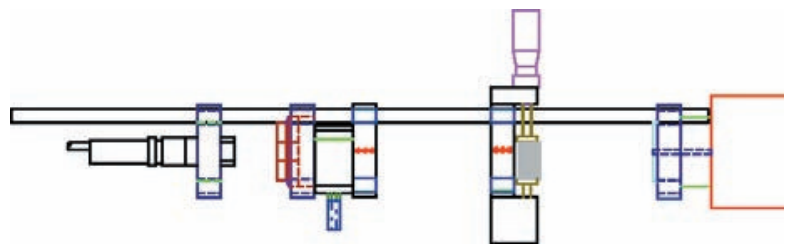

Fig. 2 Planta de la celda de sedimentación.

Por su parte, la fotografía que se presenta en la figura $N^{\circ} 3$ muestra una vista de la celda de sedimentación ya construida, destacando la cubeta, el emisor láser y el fotosensor, además del filtro gris que se empleó para reducir la potencia de señal luminosa una vez que ésta traspasó la suspensión.

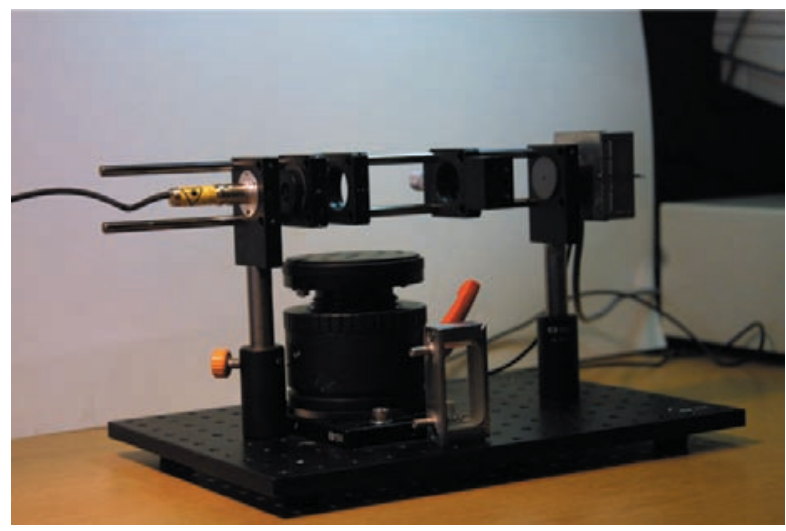

Fig. 3 Montaje de la celda de sedimentación.

Las principales características técnicas de la celda de sedimentación son las siguientes:

Puntos de medida

Posición desde superficie libre

Fuente de luz diodo láser

Diafragma Kollimatoroptik de

Diámetro del haz de luz
Filtro gris Kiel de intensidad variable

Foto sensor PIN de señal de tensión continua

Cubeta rectangular de vidrio de cuarzo de $14 \times 23 \mathrm{~mm}^{2}$ (altura×ancho)

Espesor de la cubeta

$8 \mathrm{~mm}$

Volumen de la cubeta

$6,08 \mathrm{ml}$

Software de medición

DasyLab 5.6

Las micropartículas utilizadas en este trabajo son del tipo estándares calibrados de tipo comercial, proveídas por la empresa Geltech Inc. con el código de producto S 1501. Químicamente estas micropartículas son dióxido de silicio amorfo $\left(\mathrm{SiO}_{2}\right)$, poseen una forma esférica y son rígidas. $\mathrm{Su}$ densidad media es del orden de 2.135 $\mathrm{kg} / \mathrm{m}^{3}$, con un diámetro nominal de $1,5 \mu \mathrm{m}$ y un área específica de $4,22 \mathrm{~m}^{2} / \mathrm{cm}^{3}$.

La siguiente fotografía (Figura $\mathrm{N}^{\circ} 4$ ), que fue obtenida durante los ensayos a través de un microscopio electrónico Zeiss Gemini 942, muestra la forma y el tamaño de las micropartículas de Geltech 1,5 $\mu \mathrm{m}$ utilizadas en la calibración.

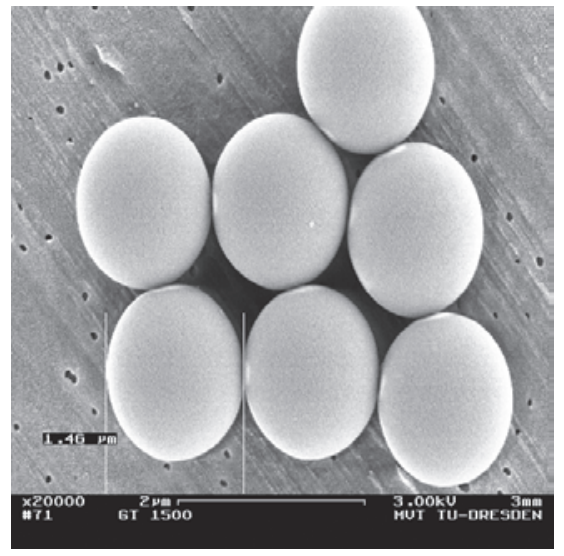

Fig. 4 Micropartículas de Geltech 1,5 $\mu \mathrm{m}$.

La suspensión se realizó con agua tridestilada, desionizada y filtrada, a la cual se le adicionó una solución de hidróxido de potasio $(\mathrm{KOH})$ a fin de que alcanzara un valor de índice de acidez $(\mathrm{pH})$ del orden de 9,2, lo que permite alcanzar un Potencial Zeta del orden $-60 \mathrm{mV}$, a fin de asegurar la estabilidad de la solución, lo que implica reducir la posibilidad de la formación de flóculos, los que interferirán con la medición.

Por otro lado, se debe evitar la contaminación por micropartículas extrañas a las que conforman la suspensión de trabajo, lo cual verificó el estado de la suspensión a través de espectrometría láser con un equipo Helos Fa. Sympatec y el análisis de las imágenes aportadas por el microscopio electrónico ya mencionado. 
La figura $\mathrm{N}^{\circ} 5$ muestra una fotografía de la suspensión ensayada a partir de una imagen del microscopio electrónico donde se aprecia la inexistencia de trazas de contaminación por partículas extrañas, entendiendo a éstas como micropartículas de un tamaño diferente del nominal.

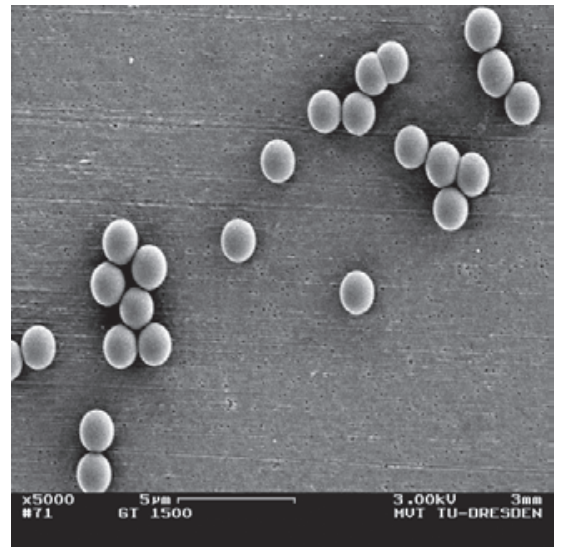

Fig. 5 Micropartículas presentes en la suspensión de calibración correspondientes a Geltech 1,5 $\mu \mathrm{m}$.

Previo a realizar cualquier prueba es necesario verificar la característica de linealidad del sensor y determinar así el valor del coeficiente de extinción, lo que se realizó experimentalmente, al determinar la magnitud que alcanza la extinción, medida para distintos valores de concentración de micropartículas en la suspensión. Estos ensayos permitieron obtener la característica de linealidad para el sistema óptico de la celda de sedimentación y que se presenta en el gráfico $\mathrm{N}^{\circ} 2$.

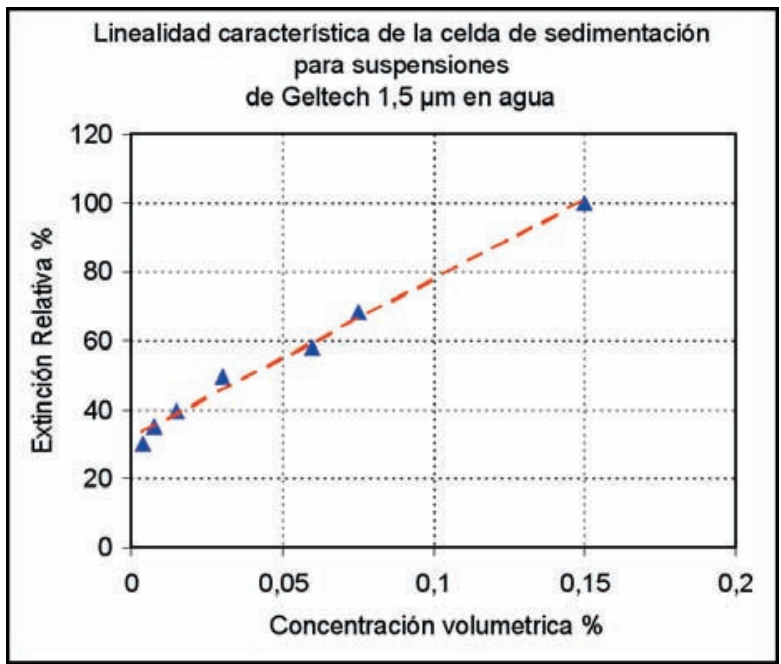

Gráfico 2 Linealidad característica del sistema óptico de la celda de sedimentación.
De esta gráfica de linealidad se desprende que el sistema óptico detecta adecuadamente los cambios de la extinción generada por las micropartículas, el que presenta un comportamiento del tipo lineal respecto de la concentración volumétrica, de modo que es posible evaluar el coeficiente de extinción para esta partícula y por lo tanto obtener mediante la aplicación de las ecuaciones (13) y (14) los valores tanto de la distribución incremental del tamaño de partícula, como de la distribución acumulativa de ésta. Para este equipo y esta partícula, el valor que alcanzó el coeficiente de extinción fue 0,1743 . En cuanto a la confiabilidad de este valor es aceptable puesto que el coeficiente de correlación de la función lineal de ajuste alcanzó a 0,99.

Conviene indicar que dados los pequeños valores que alcanza la función corrección de viscosidad para los fines prácticos de estas mediciones en particular, se despreció el valor que tomaba la función corrección de viscosidad, puesto que éste es prácticamente uno (1), por lo diluido de la suspensión. A su vez, el factor de esfericidad es unitario ya que la partícula es de forma esférica.

Como se indicó, el procedimiento de carga de la cubeta fue realizado a través de una bomba, la que trasladó la suspensión preparada para el ensayo y que previamente fue controlada, desde un vaso precipitado donde se le mantenía dispersada mediante la acción de un agitador magnético. Realizada la carga se sellaban los ductos de alimentación y descarga de la cubeta y se iniciaba el proceso de sedimentación y el programa de mediciones, los que se programaron en el sistema de adquisición de datos, a fin de realizar la lectura de la señal de tensión de corriente continua generada a partir del fotosensor, la que fue medida en $\mathrm{V}$, en intervalos de $100 \mathrm{~s}$.

Finalmente, es necesario indicar que en operación la celda de sedimentación debe utilizarse en una sala con control de temperatura ambiente y a cubierto de la radiación solar, a fin de mantener tanto el equipo como la suspensión, sin alteraciones de temperatura, ya que ésta y la energía que tiene asociada generan dos efectos que alteran la medición.

El primero de ellos está asociado a los cambios en el valor de la viscosidad del fluido (agua), los que obviamente alteran el valor de la fuerza de arrastre y con ello la velocidad de Stokes, la que es utilizada para determinar el tamaño de partícula mediante la ecuación (12).

El segundo efecto está asociado a la formación de corrientes convectivas de fluido dentro de la cubeta, las que retardarían el proceso de sedimentación, pudiendo 
arrastrar micropartículas, creando con ello zonas donde éstas chocarían o rozarían alterando así la velocidad de sedimentación y con ello la medida.

\section{RESULTADOS}

Los valores obtenidos directamente de los ensayos realizados corresponden a un conjunto de tablas de valores de tiempo y sus correspondientes valores de tensión eléctrica registrada por el fotosensor.

Considerando que los tiempos de duración de cada ensayo eran relativamente largos, ya que un ensayo típico duraba del orden de $10.000 \mathrm{~s}$, vale decir aproximadamente $2 \mathrm{hr}$. con $45 \mathrm{~min}$., y la considerable cantidad de ensayos realizados, se presentará sólo a modo de ejemplo uno de los ensayos realizados con sus correspondientes gráficos de valores obtenidos a fin de presentar la forma como se obtuvieron y la metodología de análisis de los datos experimentales. Este caso corresponde a una suspensión con una concentración volumétrica de $0,031 \%$, cuyos valores entregados sin ningún tipo de procesamiento por el sensor óptico se presentan en el gráfico $\mathrm{N}^{\circ} 3$.

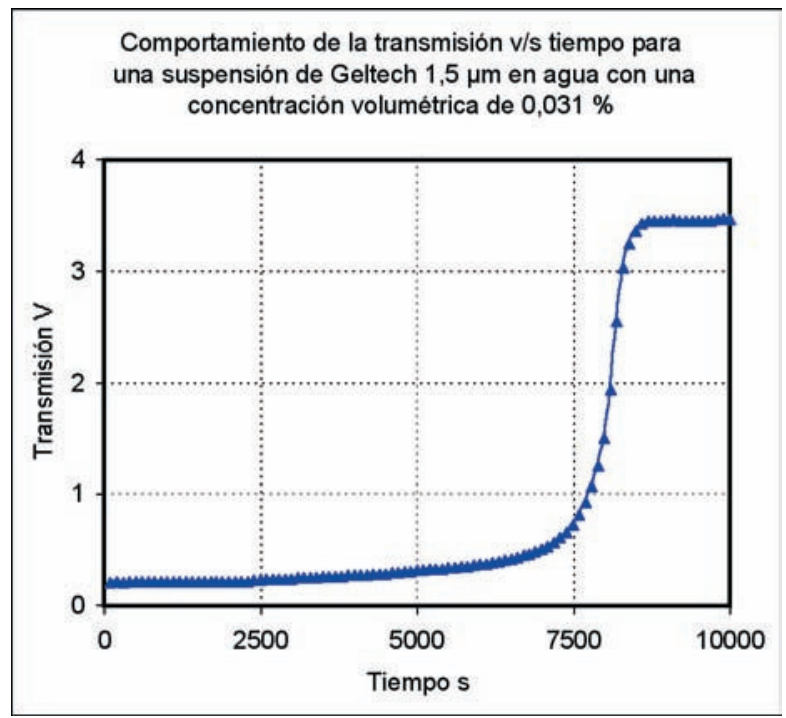

Gráfico 3 Comportamiento de la transmisión en términos de tensión eléctrica respecto del tiempo.

Los valores de la transmisión debidamente afectados por su inverso y aplicándoles la función logaritmo, es posible obtener el comportamiento de la función extinción respecto del tiempo, como se muestra en el gráfico $\mathrm{N}^{\circ} 4$.

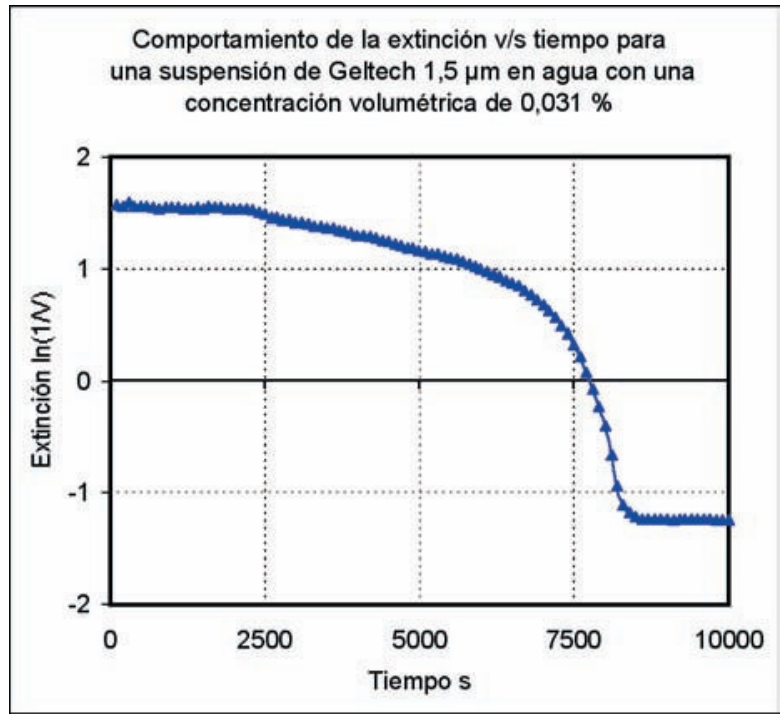

Gráfico 4 Comportamiento de la extinción en términos de tensión eléctrica respecto del tiempo.

Evaluando la velocidad de sumergimiento como la división entre la distancia desde la superficie libre de la cubeta al punto de ubicación del conjunto óptico (lásersensor) y reemplazando en la ecuación (10), se puede obtener el valor del diámetro de la partícula. Al tiempo que utilizando las ecuaciones (13) y (14), se pueden evaluar el comportamiento tanto de la concentración de tamaño incremental y acumulativa de la micropartícula. Los resultados alcanzados en este caso se presentan en el gráfico $\mathrm{N}^{\circ} 5$.

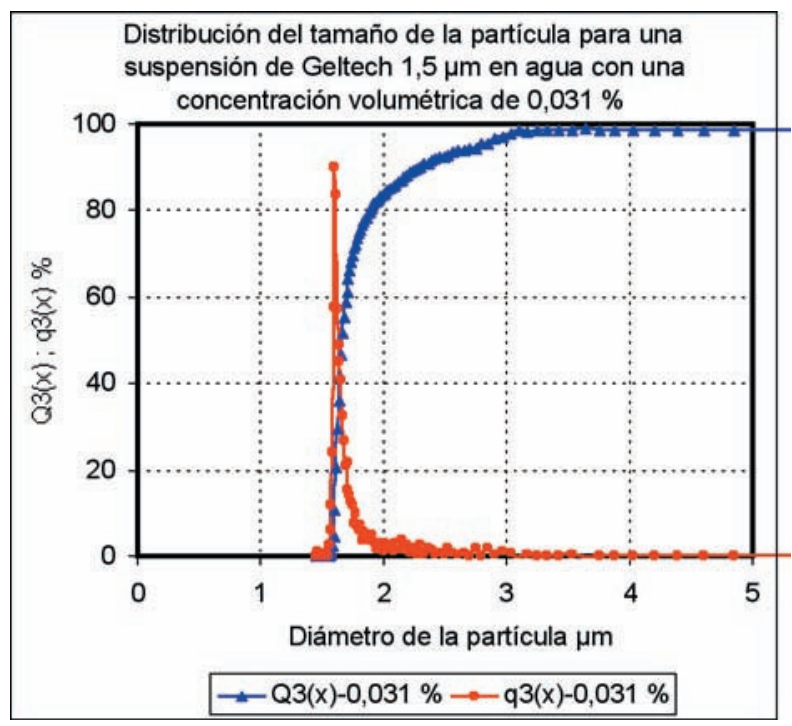

Gráfico 5 Distribución incremental y acumulativa del tamaño de micropartícula para una concentración volumétrica de $0,031 \%$. 
Se realizaron numerosos ensayos utilizando la celda de sedimentación descrita, a fin de determinar primero su capacidad de reproducibilidad en la medición, lo que se desarrolló a través de un programa de ensayos realizados en distintos días utilizando la misma suspensión.

Concluida esta primera fase, se procedió llevar a cabo un programa de ensayos tendientes a evaluar la posible influencia que en su precisión de medida tendría la concentración volumétrica de partículas, de manera que se realizó una batería de ensayos con el mismo tipo de suspensión de Geltech S 1501 en agua, pero con diferentes concentraciones volumétricas. Los valores alcanzados permitieron construir gráficas de densidad de distribución de tamaño de partícula, consistentes entre sí y con las medidas entregadas por los métodos de control, como se muestra en el gráfico $\mathrm{N}^{\circ} 6$, donde se presentan algunos de los resultados alcanzados para distintas concentraciones volumétricas estudiadas.

Por último, se estudió el comportamiento de la celda de sedimentación frente a alteraciones externas, de manera que se analizaron dos casos básicos, los que fueron el intercambio de flujo de calor entre la suspensión y el ambiente y el efecto de vibraciones mecánicas.

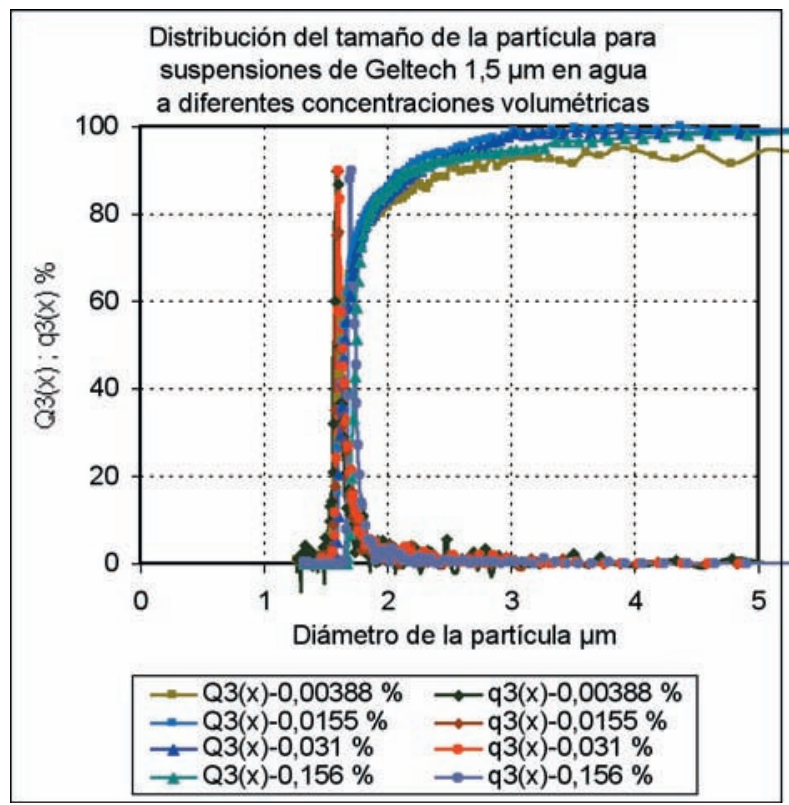

Gráfico 6 Distribución incremental y acumulativa del tamaño de micropartícula para distintas concentraciones volumétricas.

Finalmente, cabe indicar que la precisión en la medición de la distribución de tamaño de partícula mediante un método de sedimentación depende fundamentalmente de los siguientes factores:

a) La preparación de la suspensión, a fin de que ésta sea estable, lo que significa que el líquido adquiera las características de un electrolito que impida la formación de aglomerados, esto es, de grupos de partículas, las que dependiendo del potencial $\mathrm{Z}$ que posean se agrupen formando un aglomerado, el que obviamente presenta una mayor velocidad de sedimentación y genera así la aparición de una aparente segunda forma modal, entendiendo como modo al conjunto de partículas de un similar tamaño y/o diámetro.

b) La incorrecta dispersión de la suspensión, que en el evento de existir, permitirá la formación de aglomerados y de flóculos que interfieren en la medición.

c) El error que se presenta al adoptar una determinada función para la evaluación del coeficiente de arrastre que determina el valor de la fuerza de arrastre, el que es función del número de Reynolds.

d) La aproximación que implica la función viscosidad de la suspensión.

e) La imposibilidad de sostener un medio adiabático en la suspensión, lo que obviamente altera el valor de la viscosidad del fluido y puede generar corrientes convectivas en el interior de la cubeta.

f) Los errores implícitos en el manejo estadístico de los valores obtenidos, los cuales tienden a asociarse a algún tipo de ajuste como el de la distribución normal o un ajuste potencial, etc. Estos manejos de la información pueden dar lugar a la aparición de segundos modos o la desaparición de otros modos. Luego, a partir de lo anterior la posibilidad de que el método no determine la existencia de un segundo modo radica en términos prácticos al tipo de función estadística de ajuste que se aplique. Respecto a este punto cabe indicar que otros trabajos desarrollados por los autores con suspensiones bimodales y trimodales de micropartículas calibradas muestran que el método de sedimentación es preciso a la hora de determinar modos, llegando incluso a determinar familias de partículas dentro de un determinado modo [6].

Considerando los factores ya mencionados, la literatura [2], [3], [7] y [8] indica que el error que se comete al utilizar un método de sedimentación en la medición del tamaño de una micropartícula es menor a un $1 \%$ para números de Reynolds menores a 0,1 y alcanzando un $2 \%$ en el caso que los números de Reynolds sean del orden de 0,5 . 
Los resultados alcanzados por el prototipo construido, en la medición de la distribución de tamaño de partícula, se ajustan a los valores suministrados por el fabricante del material de referencia y a los valores sumistrados por los métodos de control o constatación que se utilizaron durante el desarrollo de los ensayos, los que fueron espectrometría láser, análisis de imágenes aportadas por microscopio electrónico y centrifugación analítica.

\section{CONCLUSIONES}

De los resultados alcanzados en el prototipo construido se pudo concluir que su aplicación para la evaluación de la distribución de tamaño de micropartículas fue no solo eficiente, sino que preciso. Además, presentó las ventajas de su simplicidad de construcción, bajo costo y relativa facilidad de operación, que puede ser construido en el país a costos razonables y con niveles de precisión satisfactorios.

En general, la aplicación de celdas de sedimentación para la determinación de la densidad de distribución de tamaño de partículas en general y de micropartículas en particular es un método factible de aplicarse a partículas cuyo tamaño medio supere a $1 \mu \mathrm{m}$, ya que en el caso de las micropartículas de tamaños menores a éste, no sedimentan por la sola acción del campo gravitatorio, quedando así en permanente suspensión y por lo tanto no son detectadas por el sistema de medida y no son registradas en la densidad de distribución de tamaño.

En cuanto a las desventajas del equipo, éstas radican en la larga duración de cada ensayo, lo que incrementa las posibilidades de que éste sufra alteraciones externas, tales como la formación de corrientes convectivas por efecto de calor que ingresa a la suspensión o simplemente el efecto de vibraciones mecánicas sobre la celda.

Estos problemas se superan al someter la cubeta y la suspensión que ésta contiene a un campo de fuerza centrífugo, con lo que se incrementaría notablemente la velocidad de sedimentación sin que se alteren las condiciones del escurrimiento de las micropartículas, las que continuarían sedimentándose en un régimen de velocidad de Stokes. Matemáticamente el incremento en la velocidad de sedimentación de la micropartícula producto del campo centrífugo se manifiesta al reemplazar en la ecuación (5) la aceleración de gravedad por la aceleración centrífuga que es el producto de la velocidad de rotación al cuadrado multiplicado por la posición radial de la micropartícula.
Se puede indicar para el caso de Geltech 1,5 $\mu \mathrm{m}$, que si los ensayos realizados en la celda de sedimentación se efectuaran en una centrífuga analítica a una velocidad de $600 \mathrm{rpm}$, se reduciría el tiempo de ensayo a menos de 6 min, sin que se altere la distribución incremental y acumulativa del tamaño de la micropartícula.

\section{REFERENCIAS}

[1] G.K. Batchelor, "Sedimentation in a dilute dispersion of spheres", Journal Fluid Mechanics 119: 379-408, 1982.

[2] C. Bernhardt, "Granulometrie", Deutscher Verlag für Grundstoffindustrie, 1. Auflage, 1990.

[3] H.D. Bockhardt, P. Güntzschel, A. Poetschukat, "Grundlagen der Verfahrenstechnik für Ingenieure", Deutscher Verlag für Grundstoffindustrie, 4. Auflage, 1997.

[4] A. Einstein, Eine neue Bestimmung der Moleküldimensionen, Annalen der Physik IV Folge 19: 289-306, 1906.

[5] Norma DIN 66111 sobre Métodos de Sedimentación, DIN Norme 66111 Über Sedimentationsmethoden, 1998.

[6] S. Ripperger, G. Salinas, M. Stintz, B. Wessely, "Untersuchung der Sedimentation von nanoskaligen Partikeln im Zentrifugalfeld", F\&S (Filtrieren und Separieren) 5: 230-236, 2002.

[7] H. Schubert, "Zur Theorie und zur Auslegung von Sedimentationsprozessen", Aufbereitungs-Tech. 22-8: 427- 441, 1981.

[8] H. Schubert, E. Heidenreich, F. Liepe, "Mechanische Verfahrenstechnik", Deutscher Verlag für Grundstoffindustrie, 1990.

[9] H. Sigloch, “Technische Fluidmechanik”, VDI Verlag 1. Auflage, 1996.

[10] H. Trawinski, "Die Trennung fein disperser Stoffe aus Flüssigkeiten durch Filtration und Sedimentation", Chem. Ing. Techn. 47 (7): 274-281, 1975. 